\title{
A Compact Difference Scheme for Time-Fractional Dirichlet Biharmonic Equation on Temporal Graded Meshes
}

\author{
Mingrong Cui*
}

School of Mathematics, Shandong University, Jinan 250100, Shandong, China.

Received 27 May 2020; Accepted (in revised version) 21 September 2020.

\begin{abstract}
The stability of a compact finite difference scheme on general nonuniform temporal meshes for a time fractional two-dimensional biharmonic problem is proved and graded mesh error estimates are derived. By using the Stephenson scheme for spatial derivatives discretisation, we simultaneously obtain approximate values of the gradient without any loss of accuracy. The discretisation of the Caputo derivative on graded meshes leads to a fully discrete implicit scheme. Numerical experiments support the theoretical findings and indicate that for problems with nonsmooth solutions, graded meshes have an advantage for very coarse temporal meshes.
\end{abstract}

AMS subject classifications: 65M06, 65M12, 65M15, 65N06, 65N22

Key words: Fractional biharmonic equation, nonsmooth solution, graded mesh, compact difference scheme, stability and convergence.

\section{Introduction}

Let $\Omega=(0, L)^{2}$ and $\Delta^{2}$ be the biharmonic operator,

$$
\begin{aligned}
& \Delta^{2} u(x, y, t) \\
:= & \frac{\partial^{4} u}{\partial x^{4}}(x, y, t)+\frac{\partial^{4} u}{\partial y^{4}}(x, y, t)+2 \frac{\partial^{4} u}{\partial x^{2} \partial y^{2}}(x, y, t), \quad(x, y) \in \Omega, \quad t \in(0, T] .
\end{aligned}
$$

In this work we consider the time fractional equation

$$
\begin{array}{lll}
{ }_{0}^{C} D_{t}^{\alpha} u(x, y, t)+\Delta^{2} u(x, y, t)=f(x, y, t), & (x, y) \in \Omega, & t \in(0, T], \\
u(x, y, 0)=g(x, y), & (x, y) \in \Omega, \\
u(x, y, t)=0, \quad \frac{\partial u}{\partial \vec{n}}(x, y, t)=0, & (x, y) \in \partial \Omega, \quad t \in(0, T],
\end{array}
$$

*Corresponding author. Email address: mrcui@sdu.edu.cn (M.R. Cui) 
where $\vec{n}$ is the unit outwards normal vector to the boundary $\partial \Omega$ of $\Omega$ and ${ }_{0}^{C} D_{t}^{\alpha}, 0<\alpha<1$ denotes the Caputo fractional derivative defined by

$$
{ }_{0}^{C} D_{t}^{\alpha} u(x, y, t):=\frac{1}{\Gamma(1-\alpha)} \int_{0}^{t}(t-\tau)^{-\alpha} \frac{\partial u}{\partial \tau}(x, y, \tau) d \tau .
$$

Fractional fourth-order partial differential equations arise in various applications [20]. We recall that fourth-order compact difference schemes in space, developed for one dimensional problems in $[11,24]$, have been extended to two dimensional problems in $[7,12]$. In particular, the convergence of the methods was investigated under the assumption that the exact solution is smooth and the mesh is uniform both in time and space. On the other hand, the solutions of fractional partial differential equations (FPDEs) usually are nonsmooth because the singularity of the time fractional derivative leads to a weak singularity near the initial time $t=0$. This may cause the loss of accuracy of the numerical method under consideration. Thus for the one-dimensional time-fractional diffusion equation considered in [18], Stynes and O'Riordan [23] showed that for all $(x, t) \in[0, L] \times(0, T]$, the derivatives of the corresponding solution $u$ can be estimated as follows:

$$
\begin{array}{ll}
\left|\frac{\partial^{k} u}{\partial x^{k}}(x, t)\right| \leq C & \text { for } \quad k=0,1,2,3,4, \\
\left|\frac{\partial^{l} u}{\partial t^{l}}(x, t)\right| \leq C\left(1+t^{\alpha-l}\right) & \text { for } \quad l=0,1,2 .
\end{array}
$$

It was also pointed out that the typical solutions of time-fractional reaction-diffusion problem have an initial layer at $t=0$ and the derivative $(\partial u / \partial t)(x, t)$ blows up as $t \rightarrow 0^{+}$. Yuste and Quintana-Murillo [25] generalised $L 1$ formula to non-uniform meshes. Zhang et al. [27] established the stability of the $L 1$ approximations of the Caputo derivative on nonuniform meshes and proved the convergence estimate $\mathscr{O}\left(N^{\alpha-2}+h^{4}\right)$ for a special temporal mesh.

A concise survey of finite element methods for subdiffusion problems with nonsmooth data is given in [13], and finite difference methods for nonlinear fractional differential equations based on non-uniform meshes are presented in [15]. It is worth noting that for problems with nonsmooth solutions, graded temporal partitions are more suitable (see [17] where a sharp error estimate for non-uniform meshes are proved). A time-fractional Benjamin-Bona-Mahony equation and nonlinear Korteweg-de Vries equation are, respectively, discussed in [19] and [21]. Galerkin-Legendre spectral schemes for nonlinear timespace fractional diffusion-reaction equations studied by Zaky et al. [26], use the $L 1$ scheme on graded meshes for approximation of the time fractional derivative. A compact ADI scheme for two-dimensional fractional sub-diffusion equation with Neumann boundary condition was given in [5] and time fractional Burgers' equations was discussed in [16].

Considering the fractional fourth-order problems (1.1), we note that the method of separation of variables shows that the solution also has a layer at the $t=0$. We will follow the ideas of [18] and discuss the details here. Note that since the Eq. (1.1) is linear, the 
superposition principle holds. For the homogeneous equation of (1.1), i.e. if $f(x, y, t)=0$, we seek a solution $u(x, y, t)$ in the form

$$
u(x, y, t)=T(t) \Phi(x, y), \quad(x, y, t) \in \Omega \times(0, T] .
$$

It follows that

$$
\frac{{ }_{0}^{C} D_{t}^{\alpha} T}{T}=-\frac{\Delta^{2} \Phi}{\Phi}=-\lambda
$$

This leads to the fractional differential equation

$$
{ }_{0}^{C} D_{t}^{\alpha} T(t)+\lambda T(t)=0
$$

and to the eigenvalue problem

$$
\begin{aligned}
& \Delta^{2} \Phi-\lambda \Phi=0, \quad(x, y) \in \Omega, \\
& \Phi(x, y)=0, \quad \frac{\partial \Phi}{\partial \vec{n}}(x, y)=0, \quad(x, y) \in \partial \Omega \text {. }
\end{aligned}
$$

Since $\Delta^{2}$ is a positive definite linear operator, its eigenvalues are positive. For any eigenvalue $\lambda_{i}$, the respective solution of the Eq. (1.3) has the form

$$
T_{i}(t)=c_{i} E_{\alpha}\left(-\lambda_{i} t^{\alpha}\right)
$$

where $E_{\alpha}(z)$ is the Mittag-Leffler function - i.e.

$$
E_{\alpha}(z):=\sum_{k=0}^{\infty} \frac{z^{k}}{\Gamma(\alpha k+1)} .
$$

Therefore, the solution of (1.1) has a weak singularity at $t=0$.

The paper is organised as follows. In Section 2 we use the Stephenson operator in order to discretise the spatial derivative on uniform meshes $[2,4]$. The fractional time derivative is approximated by the $L 1$-formula with graded temporal partitions. Section 3 contains the main result of this work - viz. the proof of the stability of a compact scheme on general meshes. The convergence of the method is discussed in Section 4. It is shown that for periodic problems the scheme has the fourth order accuracy in space and $\mathscr{O}\left(N^{-\min \{2-\alpha, r \alpha\}}\right)$ accuracy in time. Here, $N$ denotes the number of points in the corresponding partition of the interval $(0, T]$. The numerical experiments discussed in Section 5 show that for $r=(2-\alpha) / \alpha$, the computational error is $\mathscr{O}\left(N^{-(2-\alpha)}+h^{4}\right)$, consistent with the theoretical analysis. For nonsmooth solutions, graded meshes have an advantage for very coarse temporal meshes. Our conclusion is given in Section 6.

\section{Discretisation of Derivatives and a Compact Finite Difference Scheme}

A compact difference scheme for Eq. (1.1) on uniform temporal meshes has been discussed in [7]. For a solution with a weak singularity considered in this work, we are going 
to use graded meshes in the neighbourhoods of $t=0$. For a positive integer $N$ and $r \geq 1$, we set $t_{n}:=(n / N)^{r} T, 0 \leq n \leq N$ and $\tau_{n}=t_{n}-t_{n-1}, 1 \leq n \leq N$. If $r=1$, then the mesh is uniform. Consequently, the solution of the problem (1.1) is approximated by a finite difference scheme on a possibly graded time mesh [23].

Now we consider the partitions of the spatial domain. For a positive integer $M$ we set $h:=L / M$ and $x_{i}:=i h, y_{j}:=j h, 0 \leq i, j \leq M$. We denote by $L_{h, 0}^{2}$ the space of sequences $\left\{U_{i j}\right\}, 0 \leq i, j \leq M$ with the zero boundary conditions $U_{i j}=0,\{i, j\} \in\{0, M\}$.

Let $u\left(x_{i}, y_{j}, t_{n}\right)$ denote the exact solution value at the point $\left(x_{i}, y_{j}, t_{n}\right)$ and $U_{i j}^{n}$ the solution of the difference scheme obtained below at the same mesh point. We first consider the approximation of spatial derivatives. The Hermitian gradient $(V, W) \in\left(L_{h, 0}^{2}\right)^{2}$ has the form

$$
\left(I+\frac{h^{2}}{6} \delta_{x}^{2}\right) V_{i j}^{n}=\Delta_{x} U_{i j}^{n}, \quad\left(I+\frac{h^{2}}{6} \delta_{y}^{2}\right) W_{i j}^{n}=\Delta_{y} U_{i j}^{n}, \quad 1 \leq i, j \leq M-1,
$$

where $I$ is the identity operator and $\Delta_{x}, \delta_{x}^{2}, \Delta_{y}, \delta_{y}^{2}$ are the spatial difference operators on mesh functions defined by

$$
\begin{array}{ll}
\Delta_{x} U_{i j}^{n}=\frac{1}{2 h}\left(U_{i+1, j}^{n}-U_{i-1, j}^{n}\right), & \delta_{x}^{2} V_{i j}^{n}=\frac{1}{h^{2}}\left(V_{i-1, j}^{n}-2 V_{i j}^{n}+V_{i+1, j}^{n}\right), \\
\Delta_{y} U_{i j}^{n}=\frac{1}{2 h}\left(U_{i, j+1}^{n}-U_{i, j-1}^{n}\right), & \delta_{y}^{2} W_{i j}^{n}=\frac{1}{h^{2}}\left(W_{i, j-1}^{n}-2 W_{i j}^{n}+W_{i, j+1}^{n}\right) .
\end{array}
$$

The mesh functions $V_{i j}^{n}$ and $W_{i j}^{n}$ are, respectively, the approximations of the components $(\partial u / \partial x)\left(x_{i}, y_{j}, t^{n}\right)$ and $(\partial u / \partial y)\left(x_{i}, y_{j}, t^{n}\right)$ of the gradient vector $\nabla u_{i j}$. We also consider the operators $\tilde{\delta}_{x}^{4}$ and $\tilde{\delta}_{y}^{4}$ defined by

$$
\tilde{\delta}_{x}^{4} U_{i j}^{n}=\frac{12}{h^{2}}\left(\Delta_{x} V_{i j}^{n}-\delta_{x}^{2} U_{i j}^{n}\right), \quad \tilde{\delta}_{y}^{4} U_{i j}^{n}=\frac{12}{h^{2}}\left(\Delta_{y} W_{i j}^{n}-\delta_{y}^{2} U_{i j}^{n}\right) .
$$

Such approximations of the fourth-order derivatives of $u^{n}$ at $\left(x_{i}, y_{j}\right)$ are called the Stephenson' scheme [22]. It is known that

$$
\tilde{\delta}_{x}^{4} u_{i j}^{n}=\left.\frac{\partial^{4} u}{\partial x^{4}}\right|_{i j} ^{n}+\mathscr{O}\left(h^{4}\right), \quad \tilde{\delta}_{y}^{4} u_{i j}^{n}=\left.\frac{\partial^{4} u}{\partial y^{4}}\right|_{i j} ^{n}+\mathscr{O}\left(h^{4}\right) .
$$

At the interior points $\left(x_{i}, y_{j}\right), 1 \leq i, j \leq M-1$, the difference scheme is constructed following the ideas of [9]. We begin with the discretisation of the operator $\Delta^{2}$. For the corresponding stationary problem, the biharmonic equation $\Delta^{2} u=f$ can be approximated with the fourth order accuracy as

$$
\tilde{\Delta}_{h}^{2} u_{i j}:=\tilde{\delta}_{x}^{4}\left(I-\frac{h^{2}}{6} \delta_{y}^{2}\right) u_{i j}+\tilde{\delta}_{y}^{4}\left(I-\frac{h^{2}}{6} \delta_{x}^{2}\right) u_{i j}+2 \delta_{x}^{2} \delta_{y}^{2} u_{i j}=f_{i j}
$$

where $\tilde{\delta}_{x}^{4}$ and $\tilde{\delta}_{y}^{4}$ are the above defined one-dimensional Stephenson operators. We note that the addition of the correction term is aimed to improve the order of the local truncations from two to four - cf. [2,3]. The time-fractional derivative is approximated by the 
classical $L 1$ formula - i.e.

$$
\begin{aligned}
{ }_{0}^{C} D_{t}^{\alpha} u\left(x_{i}, y_{j}, t_{n}\right) & :=\frac{1}{\Gamma(1-\alpha)} \sum_{l=0}^{n-1} \int_{t_{l}}^{t_{l+1}}\left(t_{n}-\tau\right)^{-\alpha} \frac{\partial u}{\partial \tau}\left(x_{i}, y_{j}, \tau\right) d \tau \\
& \approx \frac{1}{\Gamma(1-\alpha)} \sum_{l=0}^{n-1} \frac{u_{i j}^{l+1}-u_{i j}^{l}}{\tau_{l+1}} \int_{t_{l}}^{t_{l+1}}\left(t_{n}-\tau\right)^{-\alpha} d \tau \\
& =\frac{1}{\Gamma(2-\alpha)} \sum_{l=0}^{n-1} \frac{u_{i j}^{l+1}-u_{i j}^{l}}{\tau_{l+1}}\left[\left(t_{n}-t_{l}\right)^{1-\alpha}-\left(t_{n}-t_{l+1}\right)^{1-\alpha}\right] \\
& =: D^{\alpha} u_{i j}^{n} .
\end{aligned}
$$

Set

$$
d_{n, l}=\frac{\left(t_{n}-t_{n-l}\right)^{1-\alpha}-\left(t_{n}-t_{n-l+1}\right)^{1-\alpha}}{\tau_{n-l+1}}, \quad 1 \leq l \leq n .
$$

Since $d_{n, 1}=\tau_{n}^{-\alpha}$, we rewrite $D^{\alpha} u_{i j}^{n}$ as

$$
D^{\alpha} u_{i j}^{n}=\frac{1}{\Gamma(2-\alpha)}\left[d_{n, 1} u_{i j}^{n}-\sum_{l=1}^{n-1}\left(d_{n, l}-d_{n, l+1}\right) u_{i j}^{n-l}-d_{n, n} u_{i j}^{0}\right] .
$$

Using (2.3) and (2.5), we can introduce the following fully discrete compact finite difference scheme for the problem (1.1).

Scheme I. Find $\left\{U_{i j}^{n}\right\} \in L_{h, 0}^{2}, 0 \leq i, j \leq M, 0 \leq n \leq N$ such that

$$
\begin{array}{lll}
D^{\alpha} U_{i j}^{n}+\tilde{\Delta}_{h}^{2} U_{i j}^{n}=f_{i j}^{n}, & 1 \leq i, j \leq M-1, & 1 \leq n \leq N, \\
U_{i j}^{0}=g_{i j}, & 1 \leq i, j \leq M-1, & \\
U_{0, j}^{n}=U_{M, j}^{n}=U_{i, 0}^{n}=U_{i, M}^{n}=0, & 0 \leq i, j \leq M, & 1 \leq n \leq N .
\end{array}
$$

Having determined the unknowns $U_{i j}$, we can derive $\left\{V_{i j}^{n}\right\}$ and $\left\{W_{i j}^{n}\right\}$ from the systems (2.1) with the boundary condition $V_{i j}^{n}=W_{i j}^{n}=0$ for mesh points $\left(x_{i}, y_{j}\right) \in \partial \Omega$. Let us write the matrix representation of Scheme I. Introducing $(M-1) \times(M-1)$ matrices $\mathbf{K}, \mathbf{P}$ and $\mathbf{T}$,

$$
\mathbf{K}:=\left(\begin{array}{ccccc}
0 & 1 & & & \\
-1 & 0 & 1 & & \\
& \ddots & \ddots & \ddots & \\
& & -1 & 0 & 1 \\
& & & -1 & 0
\end{array}\right), \quad \mathbf{P}:=\left(\begin{array}{ccccc}
4 & 1 & & & \\
1 & 4 & 1 & & \\
& \ddots & \ddots & \ddots & \\
& & 1 & 4 & 1 \\
& & & 1 & 4
\end{array}\right), \quad \mathbf{T}:=6 \mathbf{I}-\mathbf{P}
$$

we write the bidimensional Hermitian gradient as

$$
\mathbf{V}=\frac{3}{h}\left(\mathbf{I} \otimes \mathbf{P}^{-1} \mathbf{K}\right) \mathbf{U}, \quad \mathbf{W}=\frac{3}{h}\left(\mathbf{P}^{-1} \mathbf{K} \otimes \mathbf{I}\right) \mathbf{U},
$$


and the mixed derivative $\delta_{x}^{2} \delta_{y}^{2}$ as

$$
\delta_{x}^{2} \delta_{y}^{2}=\frac{1}{h^{4}} \mathbf{T} \otimes \mathbf{T}
$$

Moreover, the fourth order difference operators approximating $\partial^{4} u / \partial x^{4}$ and $\partial^{4} u / \partial y^{4}$ have the form

$$
\tilde{\delta}_{x}^{4}=\frac{12}{h^{2}} \mathbf{I} \otimes\left(\frac{3}{2 h^{2}} \mathbf{K} \mathbf{P}^{-1} \mathbf{K}+\frac{1}{h^{2}} \mathbf{T}\right), \quad \tilde{\delta}_{y}^{4}=\frac{12}{h^{2}}\left(\frac{3}{2 h^{2}} \mathbf{K} \mathbf{P}^{-1} \mathbf{K}+\frac{1}{h^{2}} \mathbf{T}\right) \otimes \mathbf{I} .
$$

Let us recall $-\mathrm{cf}$. $[8,10]$, that the tensor (Kronecker) product $A \otimes B$ of matrices $A \in R^{m, n}$ and $B \in \mathbb{R}^{p, q}$ is a matrix in $\mathbb{R}^{m \times p, n \times q}$ defined by

$$
A \otimes B=\left(\begin{array}{llll}
a_{1,1} B & a_{1,2} B & \cdots & a_{1, n} B \\
a_{2,1} B & a_{2,2} B & \cdots & a_{2, n} B \\
\vdots & \vdots & \ldots & \vdots \\
a_{m, 1} B & a_{m, 2} B & \cdots & a_{m, n} B
\end{array}\right) .
$$

We also will write $\left\{U_{i, j}\right\}$ for the column vector

$$
\mathbf{U}=\left(U_{1,1}, \cdots, U_{1, M-1}, U_{2,1}, \cdots, U_{2, M-1}, \cdots ; U_{M-1,1}, \cdots, U_{M-1, M-1}\right)^{T} \in \mathbb{R}^{(M-1)^{2}},
$$

and consider the vectors

$$
v_{1}:=(a-b)^{1 / 2} \mathbf{P}^{-1}\left(\frac{\sqrt{2}}{2} e_{1}-\frac{\sqrt{2}}{2} e_{M-1}\right), \quad v_{2}:=(a+b)^{1 / 2} \mathbf{P}^{-1}\left(\frac{\sqrt{2}}{2} e_{1}+\frac{\sqrt{2}}{2} e_{M-1}\right)
$$

with the constants

$$
a=2\left(2-e_{1}^{T} \mathbf{P}^{-1} e_{1}\right), \quad b=2 e_{M-1}^{T} \mathbf{P}^{-1} e_{1}
$$

and the column vectors $e_{1}=(1,0, \cdots, 0)^{T}, e_{M-1}=(0, \cdots, 0,1)^{T}$ in $\mathbb{R}^{M-1}$. Using the representations (2.8) and (2.9) and vectors $v_{1}, v_{2}$, we write the difference operator $\tilde{\Delta}_{h}^{2}$ in (2.3) in the following form - cf. [7]:

$$
\begin{aligned}
\tilde{\Delta}_{h}^{2}= & \frac{1}{h^{4}}\left[6\left(\mathbf{I}+\frac{1}{6} \mathbf{T}\right) \otimes \mathbf{P}^{-1} \mathbf{T}^{2}+6 \mathbf{P}^{-1} \mathbf{T}^{2} \otimes\left(\mathbf{I}+\frac{1}{6} \mathbf{T}\right)+2 \mathbf{T} \otimes \mathbf{T}\right] \\
& +\frac{36}{h^{4}}\left(\mathbf{I}+\frac{1}{6} \mathbf{T}\right) \otimes\left[\left(v_{1} v_{2}\right)\left(\begin{array}{c}
v_{1}^{T} \\
v_{2}^{T}
\end{array}\right)\right]+\frac{36}{h^{4}}\left[\left(\begin{array}{ll}
v_{1} & v_{2}
\end{array}\right)\left(\begin{array}{c}
v_{1}^{T} \\
v_{2}^{T}
\end{array}\right)\right] \otimes\left(\mathbf{I}+\frac{1}{6} \mathbf{T}\right) .
\end{aligned}
$$

Consequently, introducing the vectors

$$
\begin{aligned}
& \mathbf{U}^{0}=\left(g_{1,1}^{0}, \cdots, g_{1, M-1}^{0}, \cdots, g_{M-1,1}^{0}, \cdots, g_{M-1, M-1}^{0}\right)^{T}, \\
& \mathbf{A}_{n}=\tilde{\Delta}_{h}^{2}+\frac{d_{n, 1}}{\Gamma(2-\alpha)} \mathbf{I}, \\
& \mathbf{F}^{n}=\left(f_{1,1}^{n}, \cdots, f_{1, M-1}^{n}, \cdots, f_{M-1,1}^{n}, \cdots, f_{M-1, M-1}^{n}\right)^{T},
\end{aligned}
$$


one writes Scheme I as

$$
\begin{aligned}
& \mathbf{A}_{1} \mathbf{U}^{1}=\frac{d_{n, n}}{\Gamma(2-\alpha)} \mathbf{U}^{0}+\mathbf{F}^{1}, \\
& \mathbf{A}_{n} \mathbf{U}^{n}=\frac{1}{\Gamma(2-\alpha)} \sum_{l=1}^{n-1}\left(d_{n, l}-d_{n, l+1}\right) \mathbf{U}^{n-l}+\frac{1}{\Gamma(2-\alpha)} d_{n, n} \mathbf{U}^{0}+\mathbf{F}^{n}, \\
& U_{i j}^{0}=g_{i j}, \\
& U_{0, j}^{n}=U_{M, j}^{n}=U_{i, 0}^{n}=U_{i, M}^{n}=0, \quad 0 \leq i, j \leq M, \quad 1 \leq n \leq N .
\end{aligned}
$$

The approximate gradients $\left\{\mathbf{V}^{n}\right\}$ and $\left\{\mathbf{W}^{n}\right\}$ can be determined from (2.7) after finding $\left\{\mathbf{U}^{n}\right\}$.

Remark 2.1. Let us note that the case of the homogeneous boundary conditions considered in (1.1) is relatively simple since in (2.6) we only use the values $f_{i j}^{n}$ on the right hand side in the first equation of Scheme I. Therefore, the stability Theorem 3.2 shows that the solution is bounded by the initial value and by the right hand side function. In nonhomogeneous situation, one has to find a suitable function $\hat{u}$ satisfying the boundary conditions and consider new unknown function $u-\hat{u}$, which satisfies the homogeneous first Dirichlet boundary condition. In addition, the initial value $g(x, y)$ and the function $f(x, y, t)$ have to be correspondingly amended. Finding such a function $\hat{u}$ is a difficult task and we refer the reader to [3, Eq. (121)]. As was mentioned there, since on the boundary the values of the solution and its first order derivatives are known, they may be transformed to the right-hand side of the equation - i.e. certain additional terms have to be added to the right hand side $f_{i j}^{n}$ in Scheme I. However, we are not going to discuss this matter here.

\section{Stability for Nonuniform Time Meshes}

For vectors $\mathbf{v}=\left(v_{0}, v_{1}, \cdots, v_{K}\right)^{T}, \mathbf{w}=\left(w_{0}, w_{1}, \cdots, w_{K}\right)^{T}$, we define the inner product and norms by

$$
(\mathbf{v}, \mathbf{w})=h \sum_{j=0}^{K} v_{j} w_{j}, \quad\|\mathbf{v}\|_{2}=(\mathbf{v}, \mathbf{v})^{1 / 2}, \quad\|\mathbf{v}\|_{\infty}=\max _{0 \leq j \leq K}\left|v_{j}\right| .
$$

In what follows, we will drop subscript 2 in the discrete $L_{2}$-norm and write it simply as $\|\cdot\|$. To prove that the matrix $\tilde{\Delta}_{h}^{2}$ defined by (2.10) is symmetric positive definite, we use the properties of some one-dimensional operators.

Lemma 3.1 (cf. Refs. $[3,9]$ ). The symmetric positive definite operator $\tilde{\delta}_{x}^{4}$ can be written in the form

$$
\mathbf{S}=\frac{6}{h^{4}} \mathbf{P}^{-1} \mathbf{T}^{2}+\frac{36}{h^{4}}\left(v_{1} v_{1}^{T}+v_{2} v_{2}^{T}\right)
$$

where

$$
v_{1}=(a-b)^{1 / 2} \mathbf{P}^{-1}\left[\frac{\sqrt{2}}{2} e_{1}-\frac{\sqrt{2}}{2} e_{M}\right], \quad v_{2}=(a+b)^{1 / 2} \mathbf{P}^{-1}\left[\frac{\sqrt{2}}{2} e_{1}+\frac{\sqrt{2}}{2} e_{M}\right]
$$


with the constants

$$
a=2\left(2-e_{1}^{T} \mathbf{P}^{-1} e_{1}\right), \quad b=2 e_{M-1}^{T} \mathbf{P}^{-1} e_{1}
$$

and the vectors $e_{1}=(1,0, \cdots, 0)^{T}, e_{M-1}=(0, \cdots, 0,1)^{T}$.

The matrix $\mathbf{S}$ has the following property.

Lemma 3.2 (cf. Cui [7]). The matrix $\mathbf{S}$ is symmetric positive definite and there is a positive constant $c_{0}$ such that for any vector $\mathbf{v} \neq \mathbf{0}^{T}$, the inequality

$$
(\mathbf{S v}, \mathbf{v}) \geq \frac{c_{0}}{h^{4}}\|\mathbf{v}\|^{2}
$$

holds.

The operator $\tilde{\delta}_{y}^{4}$ is also symmetric positive definite. Note that the operators $\left(I-\left(h^{2} / 6\right)\right.$ $\left.\times \delta_{x}^{2}\right)$ and $\left(I-\left(h^{2} / 6\right) \delta_{y}^{2}\right)$ are symmetric positive definite, with $\delta_{x}^{2} \delta_{y}^{2}$ being symmetric semipositive definite.

Lemma 3.3. The matrix $\tilde{\Delta}_{h}^{2}$ is symmetric positive definite and there is a positive constant $c_{0}$ such that, for any vector $\mathbf{v} \neq \mathbf{0}^{T}$ the inequality

$$
\left(\tilde{\Delta}_{h}^{2} \mathbf{v}, \mathbf{v}\right) \geq \frac{2 c_{0}}{h^{4}}\|\mathbf{v}\|^{2}
$$

holds.

Lemma 3.3 can be used to prove the solvability of Scheme I.

Theorem 3.1. Scheme I has a unique solution.

Proof. The coefficient matrix of Scheme I is

$$
\mathbf{A}_{n}=\tilde{\Delta}_{h}^{2}+\frac{d_{n, 1}}{\Gamma(2-\alpha)} \mathbf{I} .
$$

Since by Lemma 3.3, the matrix $\tilde{\Delta}_{h}^{2}$ is symmetric positive definite and $d_{n, 1}=\tau_{n}^{-\alpha}>0$, the matrix $\mathbf{A}_{n}$ is invertible. Therefore, the finite difference scheme (2.6) is uniquely solvable. result.

In order to prove the stability and convergence of Scheme I, we need the following

Lemma 3.4 (cf. Stynes et al. [23]). The coefficients

$$
d_{n, l}=\frac{\left(t_{n}-t_{n-l}\right)^{1-\alpha}-\left(t_{n}-t_{n-l+1}\right)^{1-\alpha}}{\tau_{n-l+1}}
$$

satisfy the following estimates:

$$
\begin{aligned}
& d_{n, l+1} \leq d_{n, l}, \\
& (1-\alpha)\left(t_{n}-t_{n-l}\right)^{-\alpha}<d_{n, l}<(1-\alpha)\left(t_{n}-t_{n-l+1}\right)^{-\alpha} .
\end{aligned}
$$


Theorem 3.2. If $\left\{\mathbf{U}^{n}\right\}$ are the solutions of Scheme I for problem (1.1), then

$$
\left\|\mathbf{U}^{n}\right\| \leq\left\|\mathbf{U}^{0}\right\|+\Gamma(1-\alpha) \max _{1 \leq l \leq n} t_{l}^{\alpha / 2}\left\|\mathbf{F}^{l}\right\|, \quad 1 \leq n \leq N .
$$

Proof. Considering the inner products of $\mathbf{U}^{1}$ and $\mathbf{U}^{n}$, respectively, with the first and the second equations in (2.11), we obtain

$$
\left(\mathbf{A}_{n} \mathbf{U}^{n}, \mathbf{U}^{n}\right)=\frac{1}{\Gamma(2-\alpha)}\left(\sum_{l=1}^{n-1}\left(d_{n, l}-d_{n, l+1}\right) \mathbf{U}^{n-l}+d_{n, n} \mathbf{U}^{0}, \mathbf{U}^{n}\right)+\left(\mathbf{F}^{n}, \mathbf{U}^{n}\right), \quad 1 \leq n \leq N .
$$

It follows from (3.1) that

$$
\left(\mathbf{A}_{n} \mathbf{U}^{n}, \mathbf{U}^{n}\right)=\left(\tilde{\Delta}_{h}^{2} \mathbf{U}^{n}, \mathbf{U}^{n}\right)+\frac{d_{n, 1}}{\Gamma(2-\alpha)}\left(\mathbf{U}^{n}, \mathbf{U}^{n}\right) \geq\left(\frac{2 c_{0}}{h^{4}}+\frac{d_{n, 1}}{\Gamma(2-\alpha)}\right)\left\|\mathbf{U}^{n}\right\|^{2} .
$$

Noting that

$$
\sum_{l=1}^{n-1}\left(d_{n, l}-d_{n, l+1}\right)+d_{n, n}=d_{n, 1}
$$

and using Lemma 3.4, we obtain

$$
\begin{aligned}
& \left|\left(\mathbf{U}^{n-l}, \mathbf{U}^{n}\right)\right| \leq \frac{1}{2}\left(\left\|\mathbf{U}^{n-l}\right\|^{2}+\left\|\mathbf{U}^{n}\right\|^{2}\right), \quad 0 \leq l \leq n, \\
& \left|\left(\mathbf{F}^{n}, \mathbf{U}^{n}\right)\right| \leq \frac{d_{n, n}}{2 \Gamma(2-\alpha)}\left\|\mathbf{U}^{n}\right\|^{2}+\frac{\Gamma(2-\alpha)}{2 d_{n, n}}\left\|\mathbf{F}^{n}\right\|^{2}
\end{aligned}
$$

Therefore,

$$
\begin{aligned}
\left(\frac{2 c_{0}}{h^{4}}+\frac{d_{n, 1}}{2 \Gamma(2-\alpha)}\right)\left\|\mathbf{U}^{n}\right\|^{2} \leq & \frac{1}{2 \Gamma(2-\alpha)} \sum_{l=1}^{n-1}\left(d_{n, l}-d_{n, l+1}\right)\left\|\mathbf{U}^{n-l}\right\|^{2} \\
& +\frac{d_{n, n}}{2 \Gamma(2-\alpha)}\left\|\mathbf{U}^{0}\right\|^{2}+\frac{\Gamma(2-\alpha)}{2 d_{n, n}}\left\|\mathbf{F}^{n}\right\|^{2} .
\end{aligned}
$$

This yields

$$
\left\|\mathbf{U}^{n}\right\|^{2} \leq \frac{1}{d_{n, 1}}\left[\sum_{l=1}^{n-1}\left(d_{n, l}-d_{n, l+1}\right)\left\|\mathbf{U}^{n-l}\right\|^{2}+d_{n, n}\left\|\mathbf{U}^{0}\right\|^{2}\right]+\frac{\Gamma^{2}(2-\alpha)}{d_{n, 1} d_{n, n}}\left\|\mathbf{F}^{n}\right\|^{2} .
$$

Following [1], we set

$$
E_{k}:=\left\|\mathbf{U}^{0}\right\|^{2}+\Gamma^{2}(2-\alpha) \max _{1 \leq l \leq k} \frac{1}{d_{l, l}^{2}}\left\|\mathbf{F}^{l}\right\|^{2}
$$

and use the method of mathematical induction in order to prove that

$$
\left\|\mathbf{U}^{k}\right\|^{2} \leq E_{k}
$$


for all $1 \leq k \leq n$. For $k=1$ the inequality (3.5) is obviously true. Assume that the estimate (3.5) is valid for all $k \leq n-1$. It follows from (3.4) that $E_{k}$ is a nondecreasing sequence. Taking into account (3.3), one obtains

$$
\begin{aligned}
\left\|\mathbf{U}^{n}\right\|^{2} & \leq \frac{1}{d_{n, 1}}\left[\sum_{l=1}^{n-1}\left(d_{n, l}-d_{n, l+1}\right) E_{n}+d_{n, n}\left\|\mathbf{U}^{0}\right\|^{2}\right]+\frac{\Gamma^{2}(2-\alpha)}{d_{n, 1} d_{n, n}}\left\|\mathbf{F}^{n}\right\|^{2} \\
& \leq \frac{1}{d_{n, 1}}\left(d_{n, 1}-d_{n, n}\right) E_{n}+\frac{d_{n, n}}{d_{n, 1}}\left[\left\|\mathbf{U}^{0}\right\|^{2}+\frac{\Gamma^{2}(2-\alpha)}{d_{n, n}^{2}}\left\|\mathbf{F}^{n}\right\|^{2}\right] \\
& =E_{n}-\frac{d_{n, n}}{d_{n, 1}} E_{n}+\frac{d_{n, n}}{d_{n, 1}}\left[\left\|\mathbf{U}^{0}\right\|^{2}+\frac{\Gamma^{2}(2-\alpha)}{d_{n, n}^{2}}\left\|\mathbf{F}^{n}\right\|^{2}\right] \\
& \leq E_{n} .
\end{aligned}
$$

Thus the inequality (3.5) is true for all $n \leq N$. By Lemma 3.4, we have

$$
d_{l, l}^{-1}<\frac{t_{l}^{\alpha}}{1-\alpha}, \quad 1 \leq l \leq N
$$

so that the inequality (3.5) can be now written as

$$
\left\|\mathbf{U}^{n}\right\| \leq\left\|\mathbf{U}^{0}\right\|+\Gamma(2-\alpha) \max _{1 \leq l \leq n} \frac{1}{d_{l, l}}\left\|\mathbf{F}^{l}\right\| \leq\left\|\mathbf{U}^{0}\right\|+\frac{\Gamma(2-\alpha)}{1-\alpha} \max _{1 \leq l \leq n} t_{l}^{\alpha}\left\|\mathbf{F}^{l}\right\| .
$$

Remark 3.1. The proof of Theorem 3.2 shows that the stability is not restricted to graded meshes. Secondly, the stability does not depend on the spatial derivative operator, as long as it is positive definite. Hence, such stability results are valid for other equations discretised on general nonuniform meshes - e.g. for time fractional diffusion and convection-diffusion problems when the $L 1$ formula is used for the approximation of the Caputo derivative.

\section{Error Estimates for Graded Time Meshes}

In this work we present error estimates for the periodic problem only - i.e. the solution $u(x, y, t)$ of problem (1.1) is assumed to be $\Omega$-periodic. In the periodic case, all points are interior ones [2], the truncation error remains of order four for all mesh points. Therefore, the exact solution $u$ satisfies

$$
D^{\alpha} \mathbf{u}^{n}+\tilde{\Delta}_{h}^{2} \mathbf{u}^{n}=\mathbf{F}^{n}+\mathbf{R}^{n} .
$$

The vector $\mathbf{R}^{n}$ can be represented in the form

$$
\mathbf{R}^{n}=\left(D^{\alpha} \mathbf{u}^{n}-_{0}^{C} D_{t}^{\alpha} \mathbf{u}^{n}\right)+\left(\tilde{\Delta}_{h}^{2} \mathbf{u}^{n}-\Delta \mathbf{u}^{n}\right)=: \mathbf{R}_{1}^{n}+\mathbf{R}_{2}^{n},
$$

and the left-hand side terms admit the estimates

$$
\left\|\mathbf{R}_{1}^{n}\right\|_{\infty}=\mathscr{O}\left(n^{-\min \{2-\alpha, r \alpha\}}\right), \quad\left\|\mathbf{R}_{2}^{n}\right\|_{\infty}=\mathscr{O}\left(h^{4}\right),
$$

so that

$$
\left\|\mathbf{R}^{n}\right\|_{\infty}=\mathscr{O}\left(n^{-\min \{2-\alpha, r \alpha\}}\right)+\mathscr{O}\left(h^{4}\right) .
$$


Remark 4.1. The term $\left\|\mathbf{R}_{2}^{n}\right\|_{\infty}$ is estimated in [3], while the estimate for $\left\|\mathbf{R}_{1}^{n}\right\|_{\infty}$ follows from [14, Corollary 2.4].

The convergence of the compact finite difference scheme (2.6) is described by the following theorem.

Theorem 4.1. Let $u(x, y, t)$ and $\left\{U_{i j}^{n}\right\}$ be, respectively, the exact solution of the periodic problem (1.1) and the numerical solution obtained by Scheme I on the time graded mesh, and let $\mathbf{u}^{n}$ be defined on the mesh points similar to $\mathbf{U}^{n}$. If the exact solution $u$ satisfies the conditions

$$
\begin{array}{ll}
\left|\frac{\partial^{k+l} u}{\partial x^{k} \partial y^{l}}(x, y, t)\right| \leq C & \text { for } \quad 0 \leq k+l \leq 8, \\
\left|\frac{\partial^{m} u}{\partial t^{m}}(x, y, t)\right| \leq C\left(1+t^{\alpha-m}\right) & \text { for } \quad 0 \leq m \leq 2,
\end{array}
$$

then there are constants $C_{1}, C_{2}$ such that

$$
\left\|\mathbf{U}^{n}-\mathbf{u}^{n}\right\| \leq T^{\alpha}\left(C_{1} N^{-\min \{2-\alpha, r \alpha\}}+C_{2} \Gamma(1-\alpha) h^{4}\right), \quad 1 \leq n \leq N .
$$

Proof. Considering the error vector $\mathbf{e}^{n}=\left(e_{11}^{n}, e_{12}^{n}, \cdots, e_{M-1, M-1}^{n}\right)^{T}$ with the components $e_{i j}^{n}=U_{i j}^{n}-u\left(x_{i}, y_{j}, t^{n}\right)$, we have

$$
\mathbf{A}_{n} \mathbf{e}^{n}=\frac{1}{\Gamma(2-\alpha)} \sum_{l=1}^{n-1}\left(d_{n, l}-d_{n, l+1}\right) \mathbf{e}^{n-l}+\mathbf{R}^{n}, \quad 1 \leq n \leq N .
$$

It follows from the relation (4.1) that the local truncation error $\mathbf{R}^{n}$ can be estimated as

$$
\left\|\mathbf{R}^{n}\right\| \leq C_{1} n^{-\min \{2-\alpha, r \alpha\}}+C_{2} h^{4} .
$$

For the first Dirichlet problem, we have $e_{0, j}^{n}=e_{M, j}^{n}=e_{i, 0}^{n}=e_{i, M}^{n}=0,0 \leq i, j \leq M$. Therefore, the error $\left\{e_{i j}^{n}\right\}$ satisfies homogeneous boundary condition of Theorem 3.2. Since $\mathbf{e}^{0}=0$, Theorem 3.2 gives

$$
\begin{aligned}
\left\|\mathrm{e}^{n}\right\| & \leq \Gamma(1-\alpha) \max _{1 \leq l \leq n} t_{l}^{\alpha}\left\|\mathbf{R}^{l}\right\| \\
& \leq \Gamma(1-\alpha) \max _{1 \leq l \leq n} t_{l}^{\alpha}\left(C_{1} l^{-\min \{2-\alpha, r \alpha\}}+C_{2} h^{4}\right) \\
& \leq C_{1} \max _{1 \leq l \leq n} t_{l}^{\alpha} l^{-\min \{2-\alpha, r \alpha\}}+C_{2} \Gamma(1-\alpha) T^{\alpha} h^{4} .
\end{aligned}
$$

Recalling that $t_{l}=(l / N)^{r} T, l=0,1, \ldots, N$ and noting that $r \alpha-\min \{2-\alpha, r \alpha\} \geq 0$, we arrive at the estimate

$$
\begin{aligned}
\left\|\mathbf{U}^{n}-\mathbf{u}^{n}\right\| & \leq C_{1} \max _{1 \leq l \leq n}\left(\left(\frac{l}{N}\right)^{r} T\right)^{\alpha} l^{-\min \{2-\alpha, r \alpha\}}+C_{2} \Gamma(1-\alpha) T^{\alpha} h^{4} \\
& \leq C_{1} T^{\alpha} \max _{1 \leq l \leq n} l^{-\min \{2-\alpha, r \alpha\}}\left(\frac{l}{N}\right)^{r \alpha}+C_{2} \Gamma(1-\alpha) T^{\alpha} h^{4} \\
& \leq C_{1} T^{\alpha} N^{-r \alpha} \max _{1 \leq l \leq n} l^{r \alpha-\min \{2-\alpha, r \alpha\}}+C_{2} \Gamma(1-\alpha) T^{\alpha} h^{4}
\end{aligned}
$$




$$
\begin{aligned}
& \leq C_{1} T^{\alpha} N^{-r \alpha} n^{r \alpha-\min \{2-\alpha, r \alpha\}}+C_{2} \Gamma(1-\alpha) T^{\alpha} h^{4} \\
& \leq C_{1} T^{\alpha} N^{-\min \{2-\alpha, r \alpha\}}+C_{2} \Gamma(1-\alpha) T^{\alpha} h^{4},
\end{aligned}
$$

and the proof is complete.

\section{Numerical Experiments}

In this section, we test the convergence of our scheme numerically. As already mentioned, the Stephenson scheme allows to easily determine $\left\{\mathbf{V}^{n}\right\}$ and $\left\{\mathbf{W}^{n}\right\}$ if $\left\{\mathbf{U}^{n}\right\}$ are known. We estimate the errors in the following discrete $L_{2}$-norm and $W^{1,2}, W^{1, \infty}$ semi-norms:

$$
\begin{aligned}
& \left\|\mathbf{e}^{N}\right\|=\left\|\mathbf{e}^{N}\right\|_{l^{2}}=\left(h^{2} \sum_{i=1}^{M-1} \sum_{j=1}^{M-1}\left(e_{i j}^{N}\right)^{2}\right)^{1 / 2}, \\
& \left|\tilde{\mathbf{e}}^{N}\right|_{1,2}=\left(h^{2} \sum_{i=1}^{M-1} \sum_{j=1}^{M-1}\left[\left(V_{i j}^{N}-\frac{\partial u}{\partial x}\left(x_{i}, y_{j}, t_{N}\right)\right)^{2}+\left(W_{i j}^{N}-\frac{\partial u}{\partial y}\left(x_{i}, y_{j}, t_{N}\right)\right)^{2}\right]\right)^{1 / 2}, \\
& \left|\tilde{\mathbf{e}}^{N}\right|_{1, \infty}=\max _{1 \leq i, j \leq M-1}\left\{\left|V_{i j}^{N}-\frac{\partial u}{\partial x}\left(x_{i}, y_{j}, t_{N}\right)\right|,\left|W_{i j}^{N}-\frac{\partial u}{\partial y}\left(x_{i}, y_{j}, t_{N}\right)\right|\right\} .
\end{aligned}
$$

It was noted in [23] that for the fractional time diffusion equation the optimal value of $r$ satisfies the equation $2-\alpha=r \alpha$. The error term $N^{-\min \{2-\alpha, r \alpha\}}$ in Theorem 4.1 indicates that this is a good choice since the error estimate takes a simpler form — viz.

$$
\left\|\mathbf{U}^{n}-\mathbf{u}^{n}\right\| \leq T^{\alpha}\left(C_{1} N^{-(2-\alpha)}+C_{2} \Gamma(1-\alpha) h^{4}\right) .
$$

Thus, in numerical experiments, we choose $r=(2-\alpha) / \alpha$ and to test the numerical convergence we follow the approach of [6]. Noting the theoretical estimate (4.2), we can expect that the replacing $N$ by $\tilde{N}=\left[2^{4 /(2-\alpha)} N\right]$ and $M$ by $2 M$, will make the numerical error estimate times a factor $1 / 16$ since

$$
\begin{aligned}
\left\|\mathrm{e}\left(\tilde{N}, 2 M, t^{\tilde{N}}\right)\right\| & \approx T^{\alpha}\left(C_{1}\left(2^{4 /(2-\alpha)} N\right)^{-(2-\alpha)}+C_{2} \Gamma(1-\alpha)(h / 2)^{4}\right) \\
& =\frac{1}{16} T^{\alpha}\left(C_{1} N^{-(2-\alpha)}+C_{2} \Gamma(1-\alpha) h^{4}\right) \\
& =\frac{1}{16}\left\|\mathrm{e}\left(N, M, t^{N}\right)\right\| .
\end{aligned}
$$

Consequently, the experimental convergence order $r=r(\tau, h)$ in numerical tests is

$$
r(N, M)=\log _{2}\left(\|\mathbf{e}(N, M)\|_{*} /\left\|\mathbf{e}\left(\left[2^{4 /(2-\alpha)} N\right], 2 M\right)\right\|_{*}\right),
$$

where $\|\cdot\|_{*}$ means any of the norms $\|\cdot\|_{\infty},\|\cdot\|,|\cdot|_{1, \infty}$, or $|\cdot|_{1,2}$. As was already mentioned, we expect the experimental convergence rate $r(N, M) \approx \log _{2} 16=4$. Note that in numerical simulations we consider the cases $\alpha=0.1, \alpha=0.5$ and $\alpha=0.9$. The results are 
shown in the Tables 1-3. As noted by the referee of this work and is mentioned in [3], the reduction of the computational cost can be achieved by solving this problem on a uniform spatial grid with assistance of FFT-based technique. However, FFT is not used in our tests. Instead, the Matlab function kron is applied directly to the Kronecker product to construct the coefficient matrix. Nevertheless, we would like to thank the referee for pointing this possibility.

Example 5.1. We consider the two-dimensional problem (1.1) with the exact solution

$$
u(x, y, t)=\left(t^{\alpha}+1000 t^{3}\right) \sin ^{2}(\pi x) \sin ^{2}(\pi y) .
$$

Adding the factor 1000 in front of $t^{3}$, we enlarge the scale of the solution, so that

$$
\begin{aligned}
f(x, y, t)= & \frac{1}{4}\left(\Gamma(1+\alpha)+\frac{6000 t^{3-\alpha}}{\Gamma(4-\alpha)}\right)(1-\cos (2 \pi x)) \times(1-\cos (2 \pi y)) \\
& +4 \pi^{4}\left(t^{\alpha}+1000 t^{3}\right)(4 \cos (2 \pi x) \cos (2 \pi y)-\cos (2 \pi x)-\cos (2 \pi y)) .
\end{aligned}
$$

Take $T=1$ and $L=1$ and $r=(2-\alpha) / \alpha$ and present the corresponding errors of the compact scheme in Tables 1-3, noting that they are consistent with the theoretical estimates. For $\alpha=0.1$, the gradient of the solution $\nabla u=(\partial u / \partial x, \partial u / \partial y)=:(v, w)$, and $e_{i j}^{N}=$ $U_{i j}^{N}-u\left(x_{i}, y_{j}, t^{N}\right), e_{x, i j}^{N}=V_{i j}^{N}-v_{i j}^{N}, e_{y, i j}^{N}=W_{i j}^{N}-w_{i j}^{N}$, the errors $e^{N}, e_{x}^{N}$ and $e_{y}^{N}$ are displayed in Figs. 1-3.

We observe that for $\alpha=0.1$, the corresponding errors are smaller than for $\alpha=0.5$ and $\alpha=0.9$. Thus for smaller $\alpha$, the exact solution has lower regularity at $t=0$. In this case, the graded mesh shows its advantage. However, for smooth solutions or the ones with high regularity, uniform meshes are preferable because of smaller local truncation errors and higher convergence rates.

Table 1: Error and experiment order of convergence on graded mesh for $\alpha=0.1$.

\begin{tabular}{||c|c|c|c|c|c|c|c|c|c||}
\hline$M$ & $N$ & $\left\|\mathrm{e}^{N}\right\|_{\infty}$ & order & $\left\|\mathrm{e}^{N}\right\|$ & order & $\left|\tilde{\mathrm{e}}^{N}\right|_{1, \infty}$ & order & $\left|\tilde{\mathrm{e}}^{N}\right|_{1,2}$ & order \\
\hline 5 & 5 & 4.1172 & - & 1.8881 & - & 31.7720 & - & 22.6209 & - \\
10 & 22 & 0.2695 & 3.9333 & 0.1020 & 4.2103 & 1.9186 & 4.0496 & 1.2282 & 4.2030 \\
20 & 93 & 0.0188 & 3.8415 & 0.0072 & 3.8244 & 0.1138 & 4.0755 & 0.0690 & 4.1538 \\
40 & 398 & 0.0013 & 3.8541 & $5.1379 \mathrm{e}-4$ & 3.8087 & 0.0065 & 4.1299 & 0.0039 & 4.1451 \\
80 & 1714 & $1.0405 \mathrm{e}-4$ & 3.6432 & $4.0457 \mathrm{e}-5$ & 3.6667 & $3.4718 \mathrm{e}-4$ & 4.2267 & $2.0545 \mathrm{e}-4$ & 4.2466 \\
\hline
\end{tabular}

Table 2: Error and experiment order of convergence on graded mesh for $\alpha=0.5$.

\begin{tabular}{||c|c|c|c|c|c|c|c|c|c||}
\hline$M$ & $N$ & $\left\|\mathrm{e}^{N}\right\|_{\infty}$ & order & $\left\|\mathrm{e}^{N}\right\|$ & order & $\left|\tilde{\mathrm{e}}^{N}\right|_{1, \infty}$ & order & $\left|\tilde{\mathrm{e}}^{N}\right|_{1,2}$ & order \\
\hline 5 & 5 & 4.2495 & - & 1.9510 & - & 31.3323 & - & 22.3172 & - \\
10 & 32 & 0.2607 & 4.0268 & 0.0984 & 4.3094 & 1.9424 & 4.0117 & 1.2455 & 4.1634 \\
20 & 202 & 0.0155 & 4.0721 & 0.0059 & 4.0599 & 0.1236 & 3.9741 & 0.0755 & 4.0441 \\
40 & 1280 & $9.6123 \mathrm{e}-4$ & 4.0112 & $3.6352 \mathrm{e}-4$ & 4.0206 & 0.0077 & 4.0047 & 0.0047 & 4.0057 \\
\hline
\end{tabular}


Table 3: Error and experiment order of convergence on graded mesh for $\alpha=0.9$.

\begin{tabular}{||c|c|c|c|c|c|c|c|c|c||}
\hline$M$ & $N$ & $\left\|\mathbf{e}^{N}\right\|_{\infty}$ & order & $\left\|\mathbf{e}^{N}\right\|$ & order & $\left|\tilde{\mathbf{e}}^{N}\right|_{1, \infty}$ & order & $\left|\tilde{\mathbf{e}}^{N}\right|_{1,2}$ & order \\
\hline 5 & 5 & 4.3560 & - & 2.0017 & - & 30.9780 & - & 22.0724 & - \\
10 & 62 & 0.2621 & 4.0548 & 0.0989 & 4.3391 & 1.9388 & 3.9980 & 1.2428 & 4.1506 \\
20 & 773 & 0.0154 & 4.0891 & 0.0058 & 4.0918 & 0.1241 & 3.9656 & 0.0758 & 4.0353 \\
40 & 9615 & $9.4725 \mathrm{e}-4$ & 4.0230 & $3.5789 \mathrm{e}-4$ & 4.0185 & 0.0077 & 4.0105 & 0.0047 & 4.0115 \\
\hline
\end{tabular}

$M=1714, N=80, T=1$.

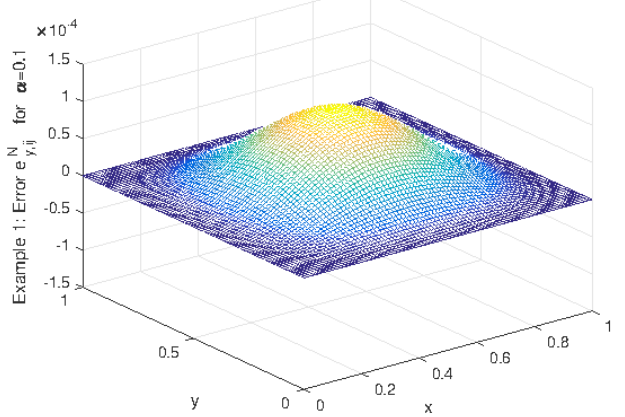

Figure 1: Error $e=U-u$ at the mesh points for $\alpha=0.1$.

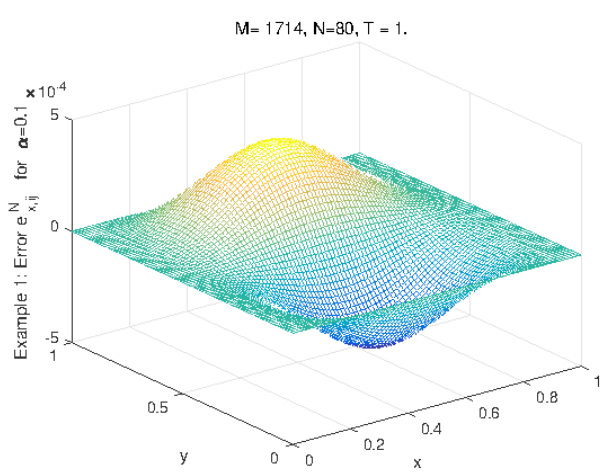

Figure 2: Error $e_{x}=V-\partial u / \partial x$ at the mesh points for $\alpha=0.1$.

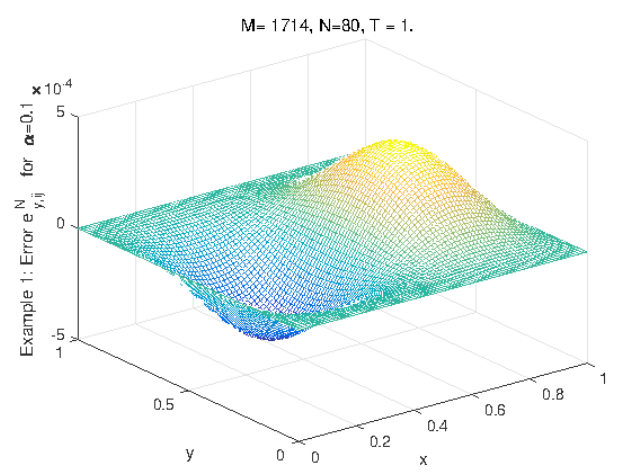

Figure 3: Error $e_{y}=W-\partial u / \partial y$ at the mesh points for $\alpha=0.1$.

Example 5.2. Let us compare the approximate solutions obtained by using graded and uniform meshes. For this, we consider the problem (1.1) having the exact solution

$$
u(x, y, t)=t^{\alpha} \sin ^{2}(\pi x) \sin ^{2}(\pi y) .
$$

The corresponding right-hand side is

$$
\begin{aligned}
f(x, y, t)= & \frac{1}{4} \Gamma(1+\alpha)(1-\cos (2 \pi x)) \times(1-\cos (2 \pi y)) \\
& +4 \pi^{4} t^{\alpha}(4 \cos (2 \pi x) \cos (2 \pi y)-\cos (2 \pi x)-\cos (2 \pi y)) .
\end{aligned}
$$


The numerical results are shown in Tables 4-5. We observe that for nonsmooth solutions and very coarse temporal mesh, the graded mesh does not have any advantage. However, for fine uniform meshes, the corresponding numerical solutions behave better than for graded meshes. Nevertheless, for graded meshes the computational error still can be estimated as $\mathscr{O}\left(N^{-(2-\alpha)}+h^{4}\right)$ for $r=(2-\alpha) / \alpha$, if the fractional time derivative is discretised by the $L 1$ method. This is in agreement to the use of the uniform meshes for smooth solutions.

Table 4: Comparison on the errors between the uniform mesh and graded mesh for $N=5$.

\begin{tabular}{||c|c|c|c|c|c|c|c||}
\hline$M$ & $N$ & $\alpha$ & $r$ & $\left\|\mathrm{e}^{N}\right\|_{\infty}$ & $\left\|\mathrm{e}^{N}\right\|$ & $\left|\tilde{\mathrm{e}}^{N}\right|_{1, \infty}$ & $\left|\tilde{\mathrm{e}}^{N}\right|_{1,2}$ \\
\hline 20 & 5 & 0.1 & 1 & $8.5497 \mathrm{e}-06$ & $3.0624 \mathrm{e}-06$ & $1.4437 \mathrm{e}-04$ & $8.9130 \mathrm{e}-05$ \\
20 & 5 & 0.1 & 19 & $1.5277 \mathrm{e}-06$ & $9.6464 \mathrm{e}-07$ & $1.7362 \mathrm{e}-04$ & $1.0841 \mathrm{e}-04$ \\
20 & 5 & 0.5 & 1 & $5.9644 \mathrm{e}-06$ & $2.0287 \mathrm{e}-06$ & $1.5210 \mathrm{e}-04$ & $9.4225 \mathrm{e}-05$ \\
20 & 5 & 0.5 & 3 & $4.3484 \mathrm{e}-06$ & $2.1890 \mathrm{e}-06$ & $1.8296 \mathrm{e}-04$ & $1.1458 \mathrm{e}-04$ \\
20 & 5 & 0.9 & 1 & $8.4825 \mathrm{e}-06$ & $3.0354 \mathrm{e}-06$ & $1.4457 \mathrm{e}-04$ & $8.9262 \mathrm{e}-05$ \\
20 & 5 & 0.9 & 1.2222 & $7.3159 \mathrm{e}-06$ & $2.5681 \mathrm{e}-06$ & $1.4806 \mathrm{e}-04$ & $9.1561 \mathrm{e}-05$ \\
\hline
\end{tabular}

Table 5: Comparison on the errors between the uniform mesh and graded mesh for $N=10$.

\begin{tabular}{||c|c|c|c|c|c|c|c||}
\hline$M$ & $N$ & $\alpha$ & $r$ & $\left\|\mathrm{e}^{N}\right\|_{\infty}$ & $\left\|\mathrm{e}^{N}\right\|$ & $\left|\tilde{\mathbf{e}}^{N}\right|_{1, \infty}$ & $\left|\tilde{\mathrm{e}}^{N}\right|_{1,2}$ \\
\hline 20 & 10 & 0.1 & 1 & $1.1491 \mathrm{e}-05$ & $4.2443 \mathrm{e}-06$ & $1.3557 \mathrm{e}-04$ & $8.3337 \mathrm{e}-05$ \\
20 & 10 & 0.1 & 19 & $1.6950 \mathrm{e}-05$ & $6.4428 \mathrm{e}-06$ & $1.1923 \mathrm{e}-04$ & $7.2605 \mathrm{e}-05$ \\
20 & 10 & 0.5 & 1 & $1.1124 \mathrm{e}-05$ & $4.0966 \mathrm{e}-06$ & $1.3667 \mathrm{e}-04$ & $8.4060 \mathrm{e}-05$ \\
20 & 10 & 0.5 & 3 & $7.3408 \mathrm{e}-06$ & $2.5781 \mathrm{e}-06$ & $1.4799 \mathrm{e}-04$ & $9.1512 \mathrm{e}-05$ \\
20 & 10 & 0.9 & 1 & $1.1462 \mathrm{e}-05$ & $4.2324 \mathrm{e}-06$ & $1.3566 \mathrm{e}-04$ & $8.3395 \mathrm{e}-05$ \\
20 & 10 & 0.9 & 1.2222 & $1.0918 \mathrm{e}-05$ & $4.0136 \mathrm{e}-06$ & $1.3728 \mathrm{e}-04$ & $8.4466 \mathrm{e}-05$ \\
\hline
\end{tabular}

\section{Conclusion}

We proved the stability of a compact finite difference scheme on general nonuniform temporal meshes for a time fractional two-dimensional biharmonic problem and derive error estimates on graded meshes. By using the Stephenson scheme for spatial derivatives discretisation, we simultaneously obtained approximate values of the gradient without any loss of accuracy. The discretisation of the Caputo derivative on graded meshes leads to a fully discrete implicit scheme. For general meshes, it is shown that this scheme is unconditionally stable and for periodic problems it converges as $T^{\alpha}\left(C_{1} N^{-\min \{2-\alpha, r \alpha\}}+C_{2} \Gamma(1-\alpha) h^{4}\right)$. The results are consistent with estimates obtained for the approximation of smooth solutions by using of uniform temporal meshes. Numerical experiments support the theoretical findings. For problems with nonsmooth solutions and when the number of the temporal mesh is small, graded meshes are more suitable than the uniform meshes. 


\section{Acknowledgements}

The author thanks the anonymous referees and the associate editor for their valuable critical comments and suggestions, which help to improve this work. Special thanks are to Professor Victor D. Didenko for his careful editing of the manuscript.

This work was supported in part by the Natural Science Foundation of Shandong Province (Grant No. ZR2014AM013), by the NSAF (Grant No. U1430101), and by NSFC (Grant No. 11471194).

\section{References}

[1] A.A. Alikhanov, A new difference scheme for the time fractional diffusion equation, J. Comput. Phys. 280, 424-438 (2015).

[2] M. Ben-Artzi, J.-P. Croisille and D. Fishelov, Convergence of a compact scheme for the pure streamfunction formulation of the unsteady Navier-Stokes system, SIAM J. Numer. Anal. 44, 1997-2024 (2006).

[3] M. Ben-Artzi, J.P. Croisille and D. Fishelov, A fast direct solver for the biharmonic problem in a rectangular grid, SIAM J. Sci. Comput. 31, 303-333 (2008).

[4] B. Bialecki, A fourth order finite difference method for the Dirichlet biharmonic problem, Numer. Algor. 61, 351-375 (2012).

[5] X.J. Cheng, H.Y. Qin and J.W. Zhang, A compact ADI scheme for two-dimensional fractional subdiffusion equation with Neumann boundary condition, Appl. Numer. Math. 156, 50-62 (2020).

[6] M.R. Cui, Convergence analysis of high-order compact alternating direction implicit schemes for the two-dimensional time fractional diffusion equation, Numer. Algor. 62, 383-409 (2013).

[7] M.R. Cui, Compact difference scheme for time-fractional fourth-order equation with first Dirichlet boundary condition, East Asian J. Appl. Math. 9, 45-66 (2019).

[8] J.W. Demmel, Applied Numerical Linear Algebra, SIAM (1997).

[9] D. Fishelov, M. Ben-Artzi, J.P. Croisille, Recent advances in the study of a fourth-order compact scheme for the one-dimensional biharmonic equations, J. Sci. Comput. 53, 55-79 (2012).

[10] G.H. Golub, C.F. Loan, Matrix Computations, John Hopkins University Press (1996).

[11] X.L. Hu, L.M. Zhang, A compact finite difference scheme for the fourth-order fractional diffusionwave system, Comput. Phys. Commun. 182, 1645-1650 (2011).

[12] C.-C. Ji, Z.-Z. Sun and Z.-P. Hao, Numerical algorithms with high spatial accuracy for the fourthorder fractional sub-diffusion equations with the first Dirichlet boundary conditions, J. Sci. Comput. 66, 1148-1174 (2016).

[13] B.T. Jin, R. Lazarov, Z. Zhou, Numerical methods for time-fractional evolution equations with nonsmooth data: A concise overview, Comput. Methods Appl. Mech. Engrg. 346, 332-358 (2019).

[14] N. Kopteva, Error analysis of the L1 method on graded and uniform meshes for a fractionalderivative problem in two and three dimensions, Math. Comput. 88, 2135-2155 (2019).

[15] C.P. Li, Q. Yi, A. Chen, Finite difference methods with non-uniform meshes for nonlinear fractional differential equations, J. Comput. Phys. 316, 614-631 (2016).

[16] L.L. Li, D.F. Li, Exact solutions and numerical study of time fractional Burgers' equations, Appl. Math. Lett. 100, 106011 (2020) .

[17] H.-L. Liao, D.F. Li, J.W. Zhang, Sharp error estimate of nonuniform L1 formula for time-fractional reaction-subdiffusion equations, SIAM J. Numer. Anal. 56, 1112-1133 (2018). 
[18] Y. Luchko, Initial-boundary-value problems for the one-dimensional time-fractional diffusion equation, Fract. Calc. Appl. Anal. 15, 141-160 (2012).

[19] P. Lyu, S. Vong, A high-order method with a temporal nonuniform mesh for a time-fractional Benjamin-Bona-Mahony equation, J. Sci. Comput. 80, 1607-1628 (2019).

[20] I. Podlubny, Fractional Differential Equations, Academic Press (1999).

[21] J.Y. Shen, Z.-Z Sun, W.R. Cao, A finite difference scheme on graded meshes for time-fractional nonlinear Korteweg-de Vries equation, Comput. Math. Appl. 361, 752-765 (2019).

[22] J.W. Stephenson, Single cell discretizations of order two and four for biharmonic problems, J. Comput. Phys. 55, 65-80 (1984).

[23] M. Stynes, E. O'Riordan, J.L. Gracia, Error analysis of a finite difference method on graded meshes for a time-fractional diffusion equation, SIAM J. Numer. Anal. 55, 1057-1079 (2017).

[24] D. Xu, W.L. Qiu, J. Guo, A compact finite difference scheme for the fourth-order time-fractional integro-differential equation with a weakly singular kernel, Numer. Methods Partial Differential Eq. 36, 439-458 (2020).

[25] S.B. Yuste, J. Quintana-Murillo, A finite difference method with non-uniform timesteps for fractional diffusion equations, Comput. Phys. Comm. 183, 2594-2600 (2012).

[26] M.A. Zaky, A.S. Hendy, J.E. Macías-Díaz, Semi-implicit Galerkin-Legendre spectral schemes for nonlinear time-space fractional diffusion-reaction equations with smooth and nonsmooth solutions, J. Sci. Comput. 82, 13 (2020).

[27] Y.N. Zhang, Z.Z. Sun, H.L. Liao, Finite difference methods for the time fractional diffusion equation on non-uniform meshes, J. Comput. Phys. 265, 195-210 (2014). 